\title{
CD133 silencing inhibits stemness properties and enhances chemoradiosensitivity in CD133-positive liver cancer stem cells
}

\author{
$\mathrm{XI} \mathrm{LAN}^{1 *}$, YONG-ZHONG WU ${ }^{2 *}$, YONG WANG ${ }^{1}$, FU-RONG WU ${ }^{2}$, \\ CHUN-BAO ZANG ${ }^{1}, \mathrm{CHUAN} \mathrm{TANG}^{3}, \mathrm{SHU}^{\mathrm{CAO}}{ }^{1}$ and SHAO-LIN LI ${ }^{1}$ \\ ${ }^{1}$ Department of Radiology, Chongqing Medical University, ${ }^{2}$ Chongqing Cancer Institute and ${ }^{3}$ Department of Neurology, \\ Yongchuan Affiliated Hospital of Chongqing Medical University, Chongqing, P.R. China
}

Received July 27, 2012; Accepted September 4, 2012

DOI: $10.3892 /$ ijmm.2012.1208

\begin{abstract}
Cancer stem cells (CSCs) are considered the source of the initial tumor formation and postoperative recurrence and metastasis. $\mathrm{CD}_{133^{+}}$cells in hepatocellular carcinoma (HCC) display cancer stem-like properties and are thought to be responsible for chemoradioresistance. To explore the functional role of CD133 in liver cancer stem cells (LCSCs), we isolated $\mathrm{CD}_{133}{ }^{+}$cells from the HCC cell line HepG2, which were tested and confirmed to be CSC-like cells in HCC, downregulated CD133 expression in HepG2-CD133+ cells by lentivirus-mediated short hairpin (shRNA) and analyzed the effects of CD133 on the modulation of stemness properties and chemoradiosensitivity in LCSCs. Our results showed that the in vitro cell proliferation, tumorsphere formation, colony formation and in vivo tumor growth in NOD/SCID mouse xenografts of LCSCs were significantly repressed after CD133 silencing. We also found that suppression of CD133 enhances the sensitivity of LCSCs to chemotherapy and radiotherapy. Knockdown of CD133 reduced G0/G1 phase cells and increased cellular apoptosis via modulation of Bcl-2 and Bax. Collectively, the stem-targeted therapy via CD133 could provide a novel strategy for the treatment of HCC.
\end{abstract}

\section{Introduction}

Hepatocellular carcinoma (HCC) is one of the most common malignancies with a poor prognosis and high recurrence rate. Although the detection rate of early HCC has increased in

Correspondence to: Dr Shao-lin Li, Department of Radiology, Chongqing Medical University, 1 Yixueyuan Road, Chongqing 400016, P.R. China

E-mail: lishaolin@cqmu.edu.cn

Dr Yong-zhong Wu, Chongqing Cancer Institute, 181 Hanyu Road, Chongqing 400030, P.R. China

E-mail: cqmdwyz@yaboo.com.cn

*Contributed equally

Key words: CD133, hepatocellular carcinoma, liver cancer stem cells, stemness properties, chemoradiosensitivity recent years and hepatectomy procedures have improved, the mortality rate remains high. Due to hepatoma cell resistance to chemotherapy and radiotherapy, the overall survival of HCC remains unsatisfactory (1). The cancer stem cell (CSC) hypothesis assumes that rare cells in tumors possess the ability of self-renewal, unlimited proliferation and pluripotency, which are the root of tumor recurrence and distant metastasis (2). Additionally, there is accumulating evidence that CSCs have stronger resistance to traditional therapies compared to cancer nonstem cells (3-9). Some reports state that functional liver cancer stem cells (LCSCs) were found in HCC cell lines (10-15), and CD133 has been used as a surface maker to isolate LCSCs $(12,16)$.

CD133 (also known as AC133 or prominin-1) is the first identified gene in the prominin family of pentaspan membrane proteins, which was originally classified as a marker of primitive hematopoietic and neural stem cells (17). Subsequent reports showed that CD133 was also expressed on some normal tissues (18-20). Furthermore, according to recent reports on the expression and distribution of the antigens, CD133 was notably detected in many types of solid tumor cells, particularly in their CSCs, such as brain tumors, renal tumors, colon carcinomas and prostate carcinomas (21-24). As a key role in maintaining the stemness properties of CSCs, CD133 possesses a feature that its expression decreases with tumor cell differentiation, making it a specific marker for isolating and identifying CSCs (25). There are reports that the expression level of CD133 has a positive correlation with advanced poorly differentiated HCC (26). Furthermore, CD133 ${ }^{+} \mathrm{HCC}$ cells have stronger proliferation capacity in vitro and tumorigenesis ability in vivo (13). However, knowledge on the functional role of CD133 in LCSCs remains preliminary.

In the present study, we isolated $\mathrm{CD} 133^{+}$cells from the human HepG2 HCC cell line and identified them as stem-like cells in HCC. Then we explored the functional role of CD133 in the modulation of stemness properties and chemoradiosensitivity in LCSCs by lentivirus-mediated CD133 silencing. Our findings strongly suggest that suppression of CD133 degrades the stemness properties and enhances the chemoradiosensitivity of LCSCs, which may provide a new strategy for future LCSC-targeted therapies and, potentially, for the therapies of other CD133-expressing types of cancer. 


\section{Materials and methods}

HCC cell lines and cell culture. The HCC cell line HepG2 was obtained from the Cell Bank of Shanghai Institutes for Biological Sciences, the Chinese Academy of Sciences (Shanghai, China). The Hep3B and SMMC-7721 HCC cell lines were obtained from the Institute of Life Sciences of Chongqing Medical University (Chongqing, China). The cell lines were routinely cultured in RPMI-1640 medium (Gibco-BRL, USA) containing 12\% heat-inactivated fetal bovine serum (Gibco-BRL) and maintained at $37^{\circ} \mathrm{C}$ in a humidified $5 \% \mathrm{CO}_{2}$ incubator.

Magnetic sorting and culture of HepG2-CD133+ cells. The cells for magnetic sorting were magnetically labeled with CD133 MicroBeads (100 $\mu \mathrm{l} / 10$ million cells) and separated on MACS MS column (were from Miltenyi Biotec). All operations were in strict accordance with the manufacturer's instructions. The purity of sorted cells was evaluated by flow cytometry and western blotting. Trypan blue staining was used for assessing the viability of sorted cells and $>90 \%$ of these cells were acceptable for the following experiments.

The fresh isolated HepG2-CD133+ cells were cultured before assay in a stem cell medium containing serum-free DMEM/F12 (1:1) medium (Gibco-BRL), $20 \mathrm{ng} / \mathrm{ml}$ epidermal growth factor (EGF), $20 \mathrm{ng} / \mathrm{ml}$ basic fibroblast growth factor (bFGF), and $20 \mathrm{ng} / \mathrm{ml}$ leukemia inhibitor factor (LIF) (all were from Miltenyi Biotec).

Flow cytometry analysis. The CD133 expression analyses were performed according to the instructions. Briefly, the fresh sorted $\mathrm{CD}_{133^{+}}$cells were incubated at $4^{\circ} \mathrm{C}$ for $30 \mathrm{~min}$ with phycoerythrin (PE)-conjugated anti-human CD133/2 following treatment with FcR Blocking Reagent kit (from Miltenyi Biotec). Isotype-matched mouse IgG2b-PE antibodies served as controls. Cell cycle and apoptosis analyses were performed using a FACSCalibur apparatus (BectonDickinson, USA) through Annexin V and propidium iodide (PI) dual staining.

Transfection of HepG2-CD133+ cells. The HIV lentiviral vector carrying interfering RNAs against CD133 (shCD133) and non-silencing RNAs (shNC) was commercially synthesized (Changsha Yingrun Biotechnologies, Inc., China). There were 3 groups: the shCD133-transfected group, the shNCtransfected group and the blank control group (untreated HepG2-CD133+ cells). Briefly, fresh sorted HepG2-CD133+ cells were plated in 6-well plates in stem cell medium. Twelve hours later, cells were transfected with shCD133 or shNC, respectively, at a multiplicity of infection (MOI) of 20. Polybrene $(5 \mu \mathrm{g} / \mathrm{ml})$ was supplemented to promote the cell transfection efficiency. Twenty-four hours post-transfection, the medium was changed to fresh culture medium.

Total-RNA isolation and RT-PCR analysis. Total-RNA was isolated from cells using TRIzol (Invitrogen, USA) according to the manufacturer's instructions. Total-RNA $(2 \mu \mathrm{l})$ was used for reverse transcription. Random hexamer primers GAPDH served as a control for RNA integrity. CD133 expression was analyzed by PCR. The primers were, CD133: forward, 5'-GAT
TCA TAC TGG TGG CTG GGT GG-3' and reverse, 5'-GCA GGT GAA GAG TGC CGT AAG T-3'; GAPDH: forward, 5'-ACC ACA GTC CAT GCC ATC AC-3' and reverse, 5'-TCC ACC ACC CTG TTG CTG TA-3'. cDNA was used in PCR reactions of 30 cycles $\left(94^{\circ} \mathrm{C} 5 \mathrm{~min}, 94^{\circ} \mathrm{C} 20 \mathrm{sec}, 55^{\circ} \mathrm{C} 25 \mathrm{sec}\right.$, $72^{\circ} \mathrm{C} 30 \mathrm{sec}$ ). PCR products were subjected to electrophoresis using $2 \%$ agarose gel and the results were analyzed by gel imaging analysis system.

Western blot analysis. Cells were washed by PBS 3 times before being harvested. Cell lysis, sample preparation, SDS-PAGE separation and electro-transferring to PVDF membrane were performed with standard methods. CD133/1 (W6B3C1) pure antibody (1:100; Miltenyi Biotec), mouse anti-GAPDH mAb (1:1,000; KFP, USA), mouse anti-Bcl-2 mAb (1:1,000; Santa Cruz Biotechnology, Inc., USA) and mouse anti-Bax mAb (1:1,000; Santa Cruz Biotechnology, Inc.) were used.

Tumorsphere-forming and colony-forming assays. For tumorsphere formation assay, cells were seeded in 6-well plates (Corning Inc., USA) in the form of single cell suspensions $(10,000$ cells/well $)$ and supported with serum-free stem cell medium as mentioned. All plates were maintained at $37^{\circ} \mathrm{C}$ in a humidified incubator and fed with $0.1 \mathrm{ml}$ medium every 3 days. Tumorspheres were observed by inverted microscopy (Olympus, Tokyo, Japan) on Days 1, 3, 5 and 7. After culturing for 7 days, serum-free stem cell medium was replaced by serum-based medium and the morphological changes of tumorspheres were observed by inverted microscopy (Olympus).

Colony-forming assay was carried out 5 days after lentivirus infection as previously described (27). Briefly, 1,000 cells were seeded in 6-well plates (Corning Inc.) and 14 days later the colonies were stained with Giemsa solution and scored if they contained $>50$ cells under an inverted microscope (Olympus).

Cell proliferation assays. Cell proliferation was examined on Days $0,1,2,3,4$ and 5 after inoculation by means of a cell proliferation assay using Cell Counting kit-8 (CCK-8) (Beyotime Institute of Biotechnology, China) according to the manufacturer's instructions. The optical density was measured using a Multiskan Spectrum (Thermo Scientific, USA) at a wavelength of $450 \mathrm{~nm}$.

Animal preparation and xenograft tumorigenicity assay. All procedures involving animals were in accordance with the institutional animal welfare guidelines of the Animal Care and Use Committee. Female 6- to 8-week-old NOD/SCID mice were purchased from the Animal Experiment Center of Chongqing Medical University (Chongqing, China) and randomly divided into 3 groups (5 mice/group). To determine the tumorigenesis ability of sorted $\mathrm{CD} 133^{+} \mathrm{HCC}$ cells in vivo, increasing number of HepG2-CD133 ${ }^{+}$cells $(100-10,000$ cells/ mouse) were suspended in $200 \mu 1$ serum-free DMEM/F2 and Matrigel (Invitrogen) mixture (1:1), and injected subcutaneously into the NOD/SCID mice. HepG2-CD133- cells were used as control. The incidence of subcutaneous tumors was recorded. Five weeks later, the grafts were separated, fixed in $10 \%$ formaldehyde solution, and embedded in paraffin for histological analysis. FACS was used to examine the expression of CD133 in the transplanted cells. The tumorigenesis 
ability of CD133-downregulated HepG2-CD133+ cells was tested based on the minimal number of HepG2-CD133+ cells which could form tumors in NOD/SCID mice.

Drug susceptibility testing. To assess the chemosensitivity of CD133 silencing cells, shCD133-transfected HepG2-CD133+ cells were seeded in 96 -well plates $(8,000$ cells/well) with serum-free stem cell medium 5 days after lentivirus infection. Cisplatin $(5 \mu \mathrm{g} / \mathrm{ml})$ and doxorubicin $(5 \mu \mathrm{g} / \mathrm{ml})$ were simultaneously added into the plates. After 24 and $48 \mathrm{~h}$, the cell growth inhibition rate (GIR) was studied by standard CCK-8 assay (28).

Radiation treatment and clonogenic assay. X-ray irradiation (IR) was delivered by an electro-linear accelerator (Varian 23EX, USA) at a dose rate of $2 \mathrm{~Gy} / \mathrm{min}$, with a surface to surface distance $(\mathrm{SSD})$ of $100 \mathrm{~cm}$. Clonogenic assay was carried out 5 days after lentivirus infection. Briefly, increasing number of cells were exposed to corresponding radiation doses (0, 2, 4, 6, 8 and $10 \mathrm{~Gy})$. Fourteen days after incubation, colonies ( $>50$ cells/colony) were counted and analyzed. Plating efficiency (PE) and survival fraction (SF) were calculated as follows: $\mathrm{PE}=$ (colony number/number of inoculated cells) $\mathrm{x} 100 \% ; \mathrm{SF}=$ colonies counted/(cells seeded x [PE/100]) (29).

In vivo study. The in vivo experiment was performed for further validation. Briefly, 5 days after lentivirus infection, $2 \times 10^{5} \mathrm{shCD} 133$-transfected HepG2-CD133 ${ }^{+}$cells were injected subcutaneously into 6- to 8-week-old NOD/SCID mice (3 mice/group). Twenty-four hours later, daily i.p. injection of cisplatin $(1.5 \mathrm{mg} / \mathrm{kg}$, for 7 days) or $4 \mathrm{~Gy}$ ionizing irradiation were administered $(29,30)$. HepG2-CD133 ${ }^{+}$cells served as blank control. Tumor size was measured using vernier calipers every week after injection for a total of 4 weeks. The volume $\left(\mathrm{mm}^{3}\right)$ was calculated by the formula: (width ${ }^{2} \mathrm{x}$ length)/2.

Statistical analysis. Statistically significant values were determined using SPSS17.0 software. Data are presented as the means \pm standard deviation (SD) and evaluated with the Student's t-test. $\mathrm{P}<0.05$ was considered to indicate statistically significant differences.

\section{Results}

CD133 expression in HCC cell lines and magnetic sorting of $\mathrm{CDI}_{33^{+}}$cells from the HepG2 cell line. Prior to sorting, CD133 expression was evaluated by flow cytometry analysis to determine the frequency of $\mathrm{CD} 133^{+}$cells in $\mathrm{HCC}$ cell lines. The results showed that $\mathrm{CD} 133^{+}$cells made up $1.16-4.37 \%$ of unsorted HepG2 cells, $0.57-0.84 \%$ of unsorted Hep3B cells and $0.21-0.56 \%$ of unsorted SMMC-7721 cells (Fig. 1A). Based on these data and our experimental task needs, we chose the HepG2 cells for the current study. After sorting by MACS, we successfully enriched a high purity of $\mathrm{CD}^{2} 33^{+}(>91 \%)$ and CD133- population from the HepG2 cell line (Fig. 1B). Then the expression level of the CD133 protein in the isolated populations was validated by western blotting. A significantly stronger expression of the $\mathrm{CD} 133$ protein was detected in the $\mathrm{CD}_{133^{+}}$population (Fig. 1C). Furthermore, we found a notable cellular morphology change between $\mathrm{CD}^{2} 33^{+}$and $\mathrm{CD} 133$ cells. The HepG2-CD133- cells grew against the wall of the flask as distributed monolayers, whereas the HepG2-CD133 ${ }^{+}$ cells grew as aggregate cell clusters (Fig. 1D).

HepG2-CD133+ cells show higher tumorsphere formation, colony-forming and proliferation ability. To verify the stemness properties of $\mathrm{CD}_{133^{+}} \mathrm{HCC}$ cells in vitro, tumorsphere formation, colony-forming and cell proliferation assays were carried out. In the sphere formation assay, we found that $\mathrm{CD}_{133^{+}}$and $\mathrm{CD} 133^{-}$cells grew in the form of suspended individual cells on the first day. As time passed, $\mathrm{CD} 133^{+}$cells grew in aggregate clusters and increased in size (Fig. 2A), whereas the CD133 cells presented aberrant cell shapes after culturing in the serum-free stem cell medium and failed to stay alive in it for more than 1 week (Fig. 2B). Forty-eight hours after replacing the serum-free stem cell medium with serum-based medium, the non-adherent tumorspheres of cells attached the bottom of the flask and grew into monolayers (Fig. 2C). Colony formation assay showed that $\mathrm{CD} 133^{+}$cells possess higher colony-forming ability than $\mathrm{CD} 133^{-}$cells. $\mathrm{CD} 133^{+}$cells formed more and larger colonies than their CD133- counterparts $(\mathrm{P}<0.001)$ (Fig. 2D and $\mathrm{E})$. In addition, we pursued the proliferative activity of $\mathrm{CD} 133^{+}$cells. The results showed that the proliferation rates of $\mathrm{CD}_{133^{+}}$cells on Days 3, 4, 5 were significantly higher than $\mathrm{CD}^{-3} 3^{-}$cells $(\mathrm{P}<0.01)$, and unsorted cells showed higher proliferation rates than $\mathrm{CD}^{2} 33^{-}$cells on Day $5(\mathrm{P}<0.05)$ (Fig. 2F and G).

Hep 2 2-CD133 ${ }^{+}$cells possess higher capacity for tumorigenicity. To validate the capacity of initiating tumors of $\mathrm{CD} 133^{+}$ HCC cells in vivo, we compared the abilities of CD133+ vs. CD133- HepG2 cells to give rise to xenografts in NOD/ SCID mice. A significant difference in tumorigenicity was found between these 2 subpopulations (Table I). As few as $1,000 \mathrm{CD} 33^{+}$cells were sufficient to form subcutaneous xenografts in 3 of 5 inoculated NOD/SCID mice 5 weeks after inoculation, and 10,000 CD133 ${ }^{+}$cells formed grafts in 5 of 5 inoculated NOD/SCID mice. However, no tumors were observed in inoculation of 10,000 CD133 cells (Fig. 3A). Hematoxylin and eosin (H\&E) staining analysis showed a highly cellular mass below the $\mathrm{CD} 133^{+}$cell injection site (Fig. 3B). Furthermore, CD133 expression was analyzed by flow cytometry to elucidate whether $\mathrm{CD} 133^{+}$cells self-renew and generate $\mathrm{CD} 133^{-}$cells in vivo. The result revealed that the grafted cells consisted of $4.30 \% \mathrm{CD}^{2} 33^{+}$and $95.72 \% \mathrm{CD} 133$ cells (Fig. 3C), which resembled the CD133 expression pattern of the original HepG2 cells.

Infection of lentivirus containing shRNA targeting CD133 in Hep $\mathrm{G} 2-\mathrm{CD} 133^{+}$cells. To assess the role of CD133 in CD133+ LCSCs, expression of CD133 in HepG2-CD133+ cells was downregulated by RNA interference (RNAi). GFP expression was observed in shCD133-transfected cells 4 days after lentivirus infection. RT-PCR results showed that the expression of CD133 mRNA was decreased by $66.45 \%$ in CD133 knockdown cells, compared to the blank control $(\mathrm{P}<0.01)$ (Fig. 4A), and western blotting results showed that the expression of the CD133 protein was significantly decreased after 7 days of lentivirus infection $(\mathrm{P}<0.01)$ (Fig. 4B). These results indicated that $\mathrm{CD} 133$ was efficiently downregulated in HepG2-CD133+ cells by lentivirus-mediated shRNA. 

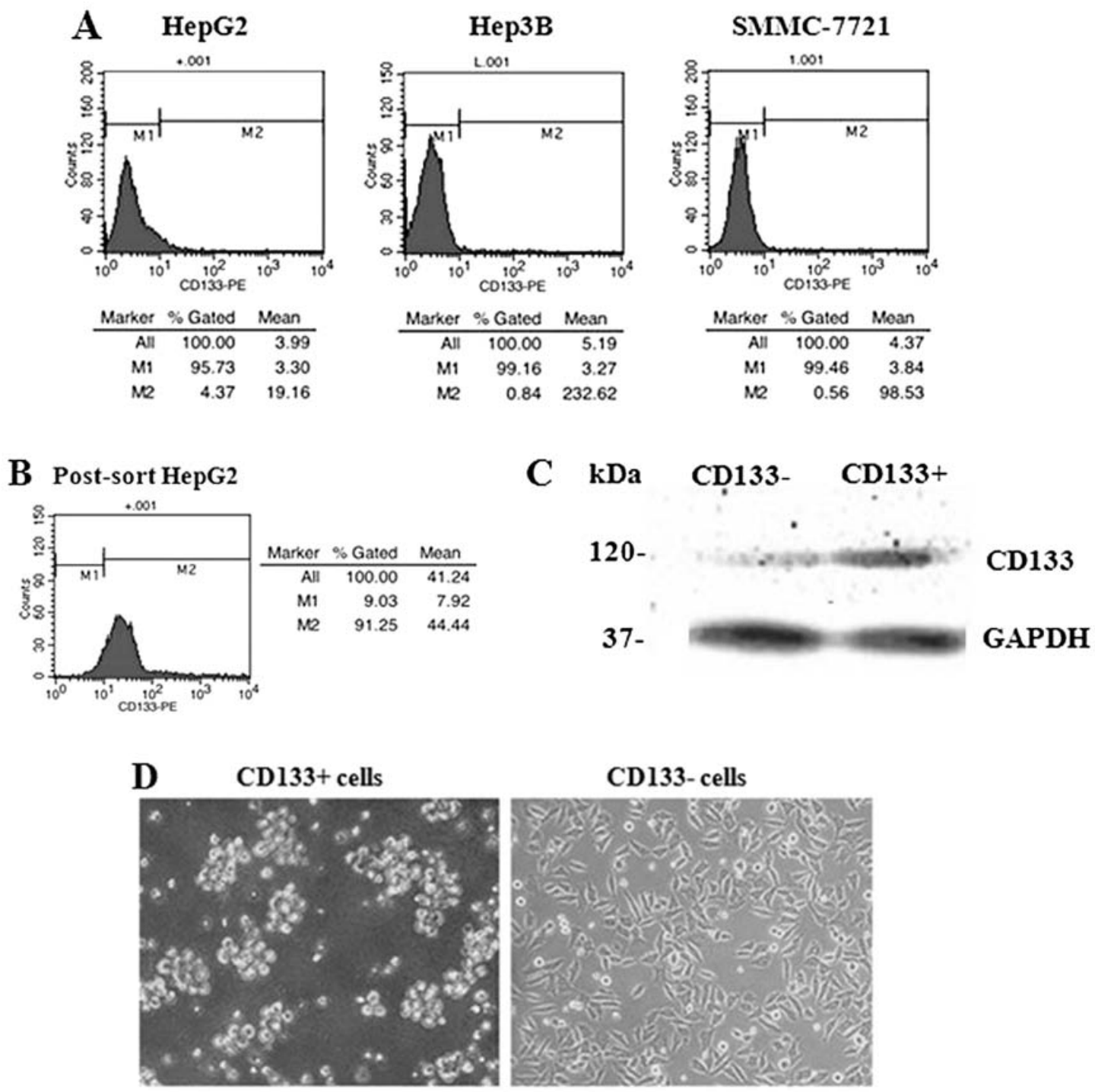

Figure 1. CD133 expression and sorting purity analysis in HCC cell lines. (A) Flow cytometry was used to analyze the expression of CD133 in HepG2, Hep3B and SMMC-7721 cells. The rates of CD133+ cells in HepG2, Hep3B and SMMC-7721 cells were, respectively, 1.16-4.37\%, 0.57-0.84\%, 0.21-0.56\%; (B) MACS sorted HepG2-CD133+ cells with a purity ranging from 82.17 to $91.25 \%$. (C) CD133 expression in HepG2 CD133 ${ }^{+}$and CD133- sorted cells was examined by western blotting. (D) The different growth patterns in $\mathrm{CD}_{133^{+}}$and $\mathrm{CD} 133^{-}$cells.

Silencing of CD133 suppresses stemness properties of

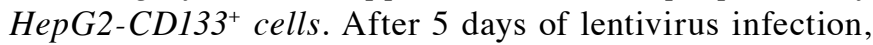
tumorsphere formation, colony-forming and proliferation assays were performed again to examine the biological changes in CD133-downregulated LCSCs. Fig. 4C shows that $\mathrm{CD}_{133^{+}}$cells transfected with shCD133 displayed a significantly reduced proliferation rate, compared to the shNC and the blank control group $(\mathrm{P}<0.01)$. Giemsa stained colonies revealed that $\mathrm{CD} 133$ downregulation resulted in a dramatic decrease in the number and size of colonies $(\mathrm{P}<0.001)$ (Fig. 4D and E). Furthermore, this shCD133-induced CD133 downregulation was accompanied by a reduced number and a smaller size of tumorspheres (Fig. 4F). Additionally, the tumorigenesis ability of CD133 silenced cells was tested and the results showed that $1,000 \mathrm{HepG} 2-\mathrm{CD} 133^{+}$cells failed to form tumor xenografts in NOD/SCID mice as they once did after CD133 downregulation. Also, 10,000 CD133downregulated cells formed tumors in 2 of 5 inoculated NOD/ SCID mice. Only as many as $1 \times 10^{5}$ CD133 downregulated cells produced tumors in 5 of $5 \mathrm{NOD} / \mathrm{SCID}$ mice 5 weeks after inoculation (Table I).

Suppression of CD133 enhances chemoradiosensitivity of Hep $\mathrm{G} 2-\mathrm{CD} 133^{+}$cells. To explore the potential role of CD133 in chemoradiosensitivity in CD133 ${ }^{+}$LCSCs, experiments in vitro and in vivo were employed. We assessed the sensitivity of the cells to cisplatin and doxorubicin. Compared with the shNC and the blank control group, both the cell GIR of cisplatin and doxorubicin in the shCD133 group were higher $(\mathrm{P}<0.01)$ (Fig. 5A and B). For radiosensitivity testing in vitro, an apparent difference in clone rate (GR) and survival fraction (SF) was observed after radiation treatment (Table II). The SF of the shCD133 group was significantly lower than the shNC and the blank control group after 14 days of incubation $(\mathrm{P}<0.01)$ (Fig. 5C). Furthermore, we pursued the chemoradiosensitivity of CD133-downregulated LCSCs in vivo. Our data showed that either cisplatin treatment or $4 \mathrm{~Gy}$ radiation treatment effectively restrained the growth speed of 

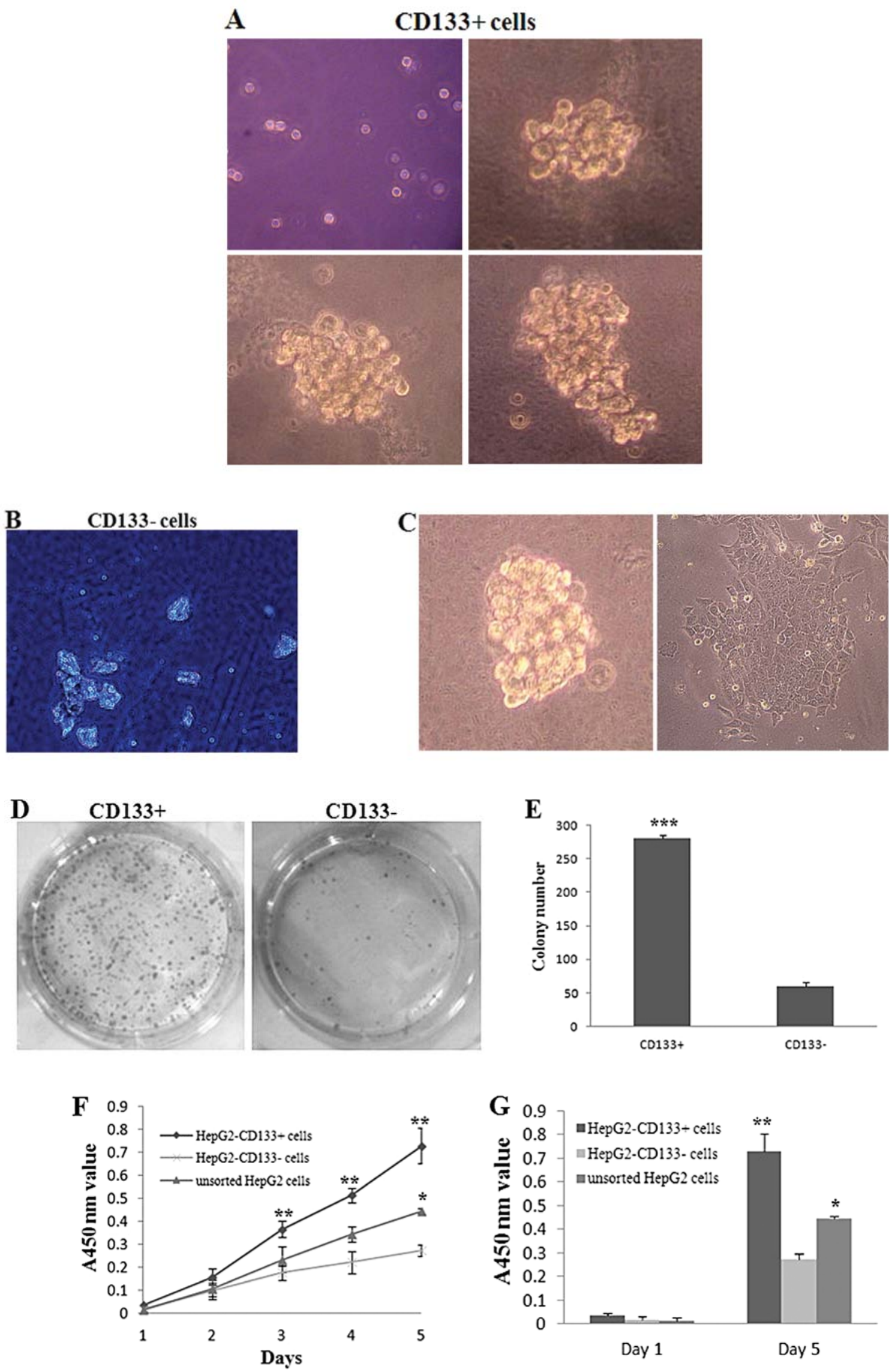

Figure 2. Characterization of CD133 ${ }^{+} \mathrm{HCC}$ cells in vitro. (A and B) Gradually increasing tumorspheres were observed in CD133 $3^{+}$cells cultured in serum-free medium, whereas CD133 cells showed aberrant cell shapes under the same cultural conditions (x200). (C) Left, a representative tumor spheroid under microscope; right, spheroids formed by $\mathrm{CD} 133^{+}$cells grew by static adherence $48 \mathrm{~h}$ after replacing serum-free stem cell medium with serum-based medium (x200). (D and E) Significant difference in number of colonies between CD133 ${ }^{+}$and CD133- cells were observed 14 days after inoculation. (F and G) CCK-8 assay showed that $\mathrm{CD} 133^{+}$cells possess much higher proliferation than $\mathrm{CD} 133{ }^{-}$cells. Data represent the means \pm SD derived from triplicate samples. ${ }^{*} \mathrm{P}<0.05,{ }^{* * *} \mathrm{P}<0.01,{ }^{* * * *} \mathrm{P}<0.001$. 

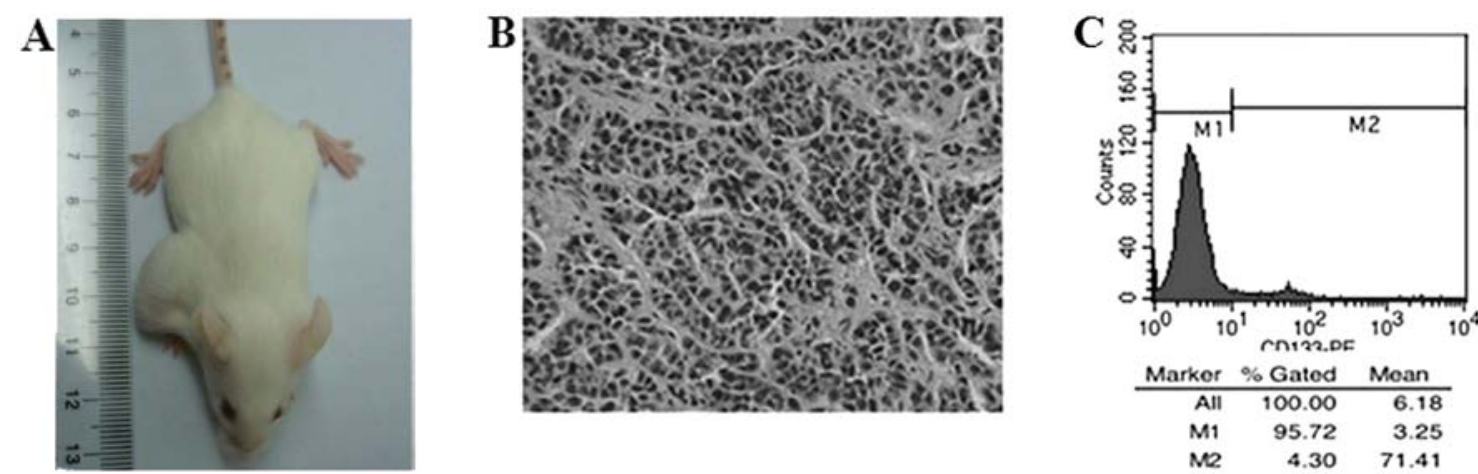

Figure 3. Tumorigenesis ability of HepG2-CD133+ cells. (A) A representative example of NOD/SCID mice injected with HepG2 CD133 ${ }^{+}$cells and CD133 cells. Bold black arrows show the tumor nodules formed by HepG2-CD133+ cells. No tumor nodules formed in the HepG2-CD133- cell inoculation site. (B) The histological feature of xenografts formed by HepG2-CD133+ cells was observed using H\&E staining (x200). (C) The xenograft tumor was applied with collagenase and CD133 expression of isolated cells was examined by flow cytometry.
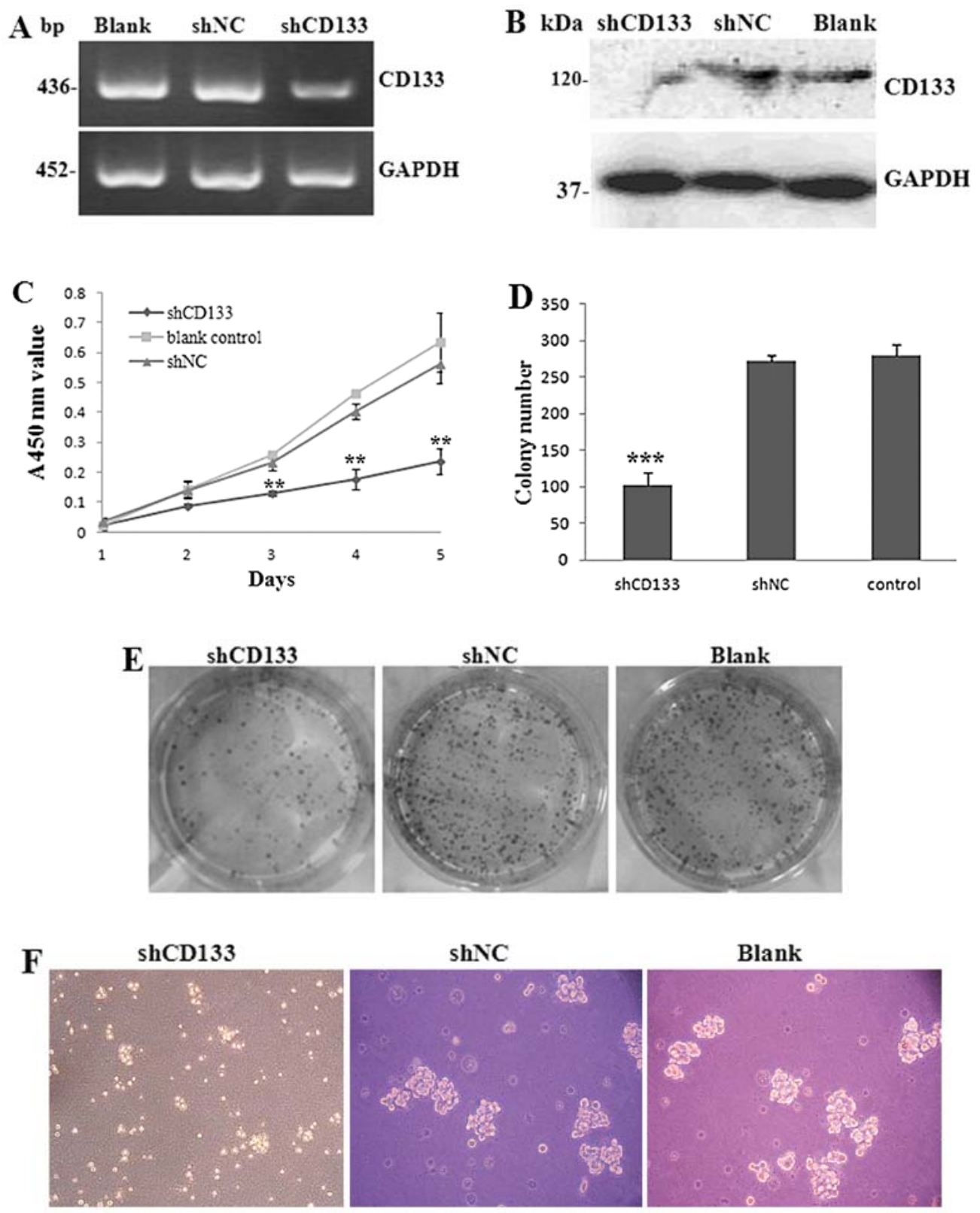

Figure 4. Silencing of CD133 suppresses stemness properties of HepG2-CD133+ cells. (A and B) The expression of CD133 mRNA and protein were significantly decreased in the shCD133 group after 7 days of RNAi $(\mathrm{P}<0.01)$. (C) The cell proliferation ability was significantly decreased in the shCD133 group. (D and E) Dramatically decreased colony number and size were observed in the shCD133 group. (F) Silencing of CD133 showed a reduced number and a smaller size of tumorspheres. Data represent the means \pm SD derived from triplicate samples. ${ }^{* *} \mathrm{P}<0.01,{ }^{* * * *} \mathrm{P}<0.001$. 
Table I. Tumorigenicity study.

No. of cells

injected/mouse

$\mathrm{CD} 133^{+} \mathrm{HCC}$ cells

CD133- HCC cells

shCD133-HCC cells

\begin{tabular}{lccc}
\hline $1 \times 10^{2}$ & $0 / 5^{\mathrm{a}}$ & $0 / 5$ & - \\
$1 \times 10^{3}$ & $3 / 5$ & $0 / 5$ & $0 / 5$ \\
$1 \times 10^{4}$ & $5 / 5$ & $0 / 5$ & $2 / 5$ \\
$1 \times 10^{5}$ & - & - & $5 / 5$
\end{tabular}

HepG2 CD133+ cells and CD133- cells were subcutaneously inoculated into 2 opposite sites of the same NOD/SCID mouse. ShCD133transfected cells were subcutaneously inoculated into the same location of NOD/SCID mice. Tumor xenografts were observed for 5 weeks after inoculation. ${ }^{a}$ Values represent the number of mice with tumors formed/number of mice inoculated. HCC, hepatocellular carcinoma.

Table II. Clone rate and survival fraction study.

\begin{tabular}{|c|c|c|c|c|c|c|c|}
\hline \multirow{2}{*}{$\begin{array}{l}\text { No. of } \\
\text { cells/well }\end{array}$} & \multirow{2}{*}{$\begin{array}{l}\text { IR dose } \\
\text { (Gy) }\end{array}$} & \multicolumn{2}{|c|}{ shCD133 } & \multicolumn{2}{|c|}{ shNC } & \multicolumn{2}{|c|}{ Blank control } \\
\hline & & CR (\%) & $\mathrm{SF}(\%)$ & CR $(\%)$ & $\mathrm{SF}(\%)$ & CR (\%) & SF (\%) \\
\hline 200 & 0 & $18.00 \pm 2.78$ & $100.00 \pm 15.47$ & $35.50 \pm 3.77$ & $101.43 \pm 10.79$ & $35.00 \pm 4.92$ & $100.00 \pm 14.07$ \\
\hline 400 & 2 & $7.50 \pm 1.40$ & $41.67 \pm 7.73$ & $31.00 \pm 2.61$ & $88.57 \pm 7.46$ & $31.58 \pm 2.65$ & $90.24 \pm 7.57$ \\
\hline 600 & 4 & $2.67 \pm 0.88$ & $14.81 \pm 4.90$ & $23.83 \pm 5.00$ & $68.10 \pm 14.29$ & $23.38 \pm 3.78$ & $66.83 \pm 10.80$ \\
\hline 800 & 6 & $1.13 \pm 0.25$ & $6.25 \pm 1.39$ & $14.25 \pm 1.19$ & $40.71 \pm 3.41$ & $15.75 \pm 1.35$ & $45.00 \pm 3.86$ \\
\hline 1,000 & 8 & $0.16 \pm 0.06$ & $0.93 \pm 0.32$ & $7.30 \pm 0.46$ & $20.86 \pm 1.31$ & $8.07 \pm 1.00$ & $23.05 \pm 2.86$ \\
\hline 1,200 & 10 & $0.06 \pm 0.05$ & $0.15 \pm 0.28$ & $1.25 \pm 0.22$ & $3.75 \pm 0.63$ & $1.50 \pm 0.44$ & $4.29 \pm 1.26$ \\
\hline
\end{tabular}

IR, X-ray irradiation; SF, survival fraction.

tumors in the shCD133 group. Four weeks later, xenografts were separated. Tumor volumes in the shCD133 group were significantly smaller than in the blank control group $(\mathrm{P}<0.01)$ (Fig. 5D and E). These results support a functional role of CD133 in LCSC chemoradioresistance. Downregulation of CD133 significantly increased the sensitivity of LCSCs to chemotherapy and radiotherapy.

Effects of CD133 downregulation on cell cycle and apoptosis. Effects of CD133 in the regulation of cell cycle and apoptosis were assessed to explore the possible mechanism of enhanced chemoradiosensitivity. The results of cell cycle analyses showed that the fresh isolated CD133+ LCSCs were mostly distributed in the static phase (G0/G1). Following downregulation of CD133 by RNAi, cells in the G0/G1 phases of the cell cycle decreased significantly, which revealed that silencing of CD133 initiated LCSC action to differentiate (Fig. 6A and B). We also measured the apoptotic cells by flow cytometry analysis. It was observed that up to $26.60 \pm 3.92 \%$ of the shCD133 group cells became apoptotic 5 days after lentivirus infection, whereas the apoptosis rate was $3.71 \pm 0.47 \%$ in the shNC group and $2.77 \pm 0.94 \%$ in the blank control group $(\mathrm{P}<0.05)$ (Fig. 6C and D). We then examined the expression of several molecules involved in cell survival regulation. Western blotting results showed that downregulation of CD133 decreased the expression level of $\mathrm{Bcl}-2$ but increased the expression level of Bax (Fig. 6E).

\section{Discussion}

The cancer stem cell (CSC) hypothesis suggests that only a small subset of cells within a tumor possess the ability to self-renew and differentiate to multiple lineages, which were defined as tumor-initiating cells (31). Studies have confirmed that CSCs exist in both hematologic and solid tumors $(32,33)$. CD133, a member of pentaspan membrane proteins encoded by the PROM1 gene, represents a marker of tumor-initiating cells in a number of human cancers (34), and has been used to isolate liver cancer stem cells (LCSCs) from hepatocellular carcinoma in recent years $(28,35)$. Therefore, taking the CSC model as a reference $(6,36)$, LCSC-targeted therapy via this marker may be an effective and curative strategy for eradicating cancer. Here, we sorted CD133 ${ }^{+}$cells from HepG 2 cells, which demonstrated many stem-like properties. We downregulated CD133 in HepG2 CD133+ cells by lentivirus-mediated RNAi and analyzed the functional role of CD133 in the modulation of stemness properties and chemoradiosensitivity in LCSCs.

Our analysis of CD133 expression in the HepG2, Hep3B and SMMC-7721 cell lines showed that HepG2 cells possess higher endogenic CD133 expression, compared to Hep3B and SMMC-7721 cells. After sorting by MACS, high-purity HepG2-CD133 ${ }^{+}$cells were obtained for subsequent experiments. In order to verify the CSC-like properties of sorted $\mathrm{CD}_{133}{ }^{+}$cells, the proliferation, tumorsphere formation, colony-forming in vitro and tumorigenicity in vivo abilities 

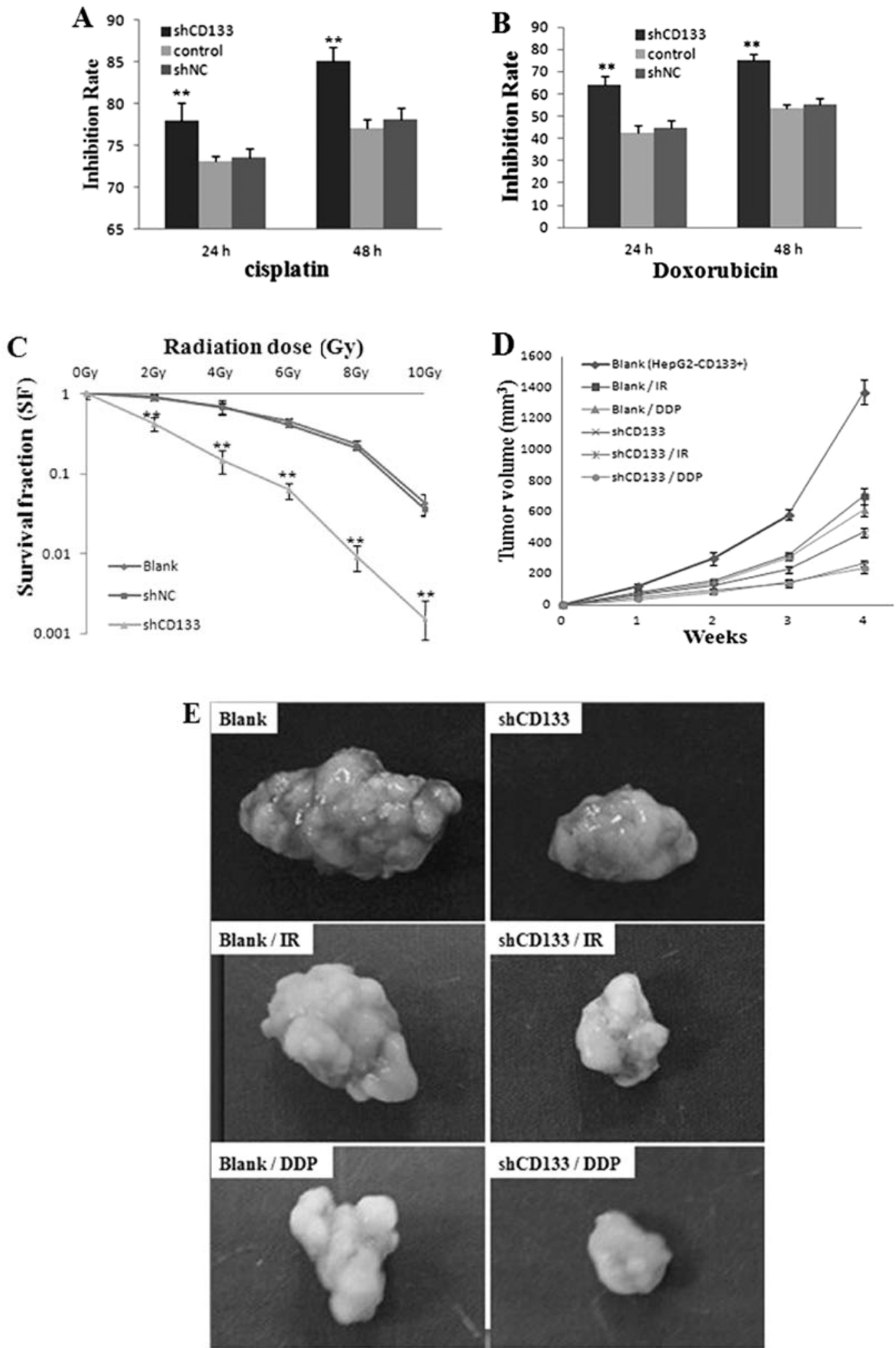

Figure 5. Suppression of CD133 enhances chemoradiosensitivity of HepG2-CD133+ cells. (A and B) Compared to the shNC and the blank control group, both the GIR of cisplatin and doxorubicin were higher in the shCD133 group. (C) After radiation treatment with doses from 0 to 10 Gy, the survival fraction in the shCD133 group was significantly lower than in the shNC and the blank control group. (D and E) The in vivo experiment showed a slower tumor growth speed and smaller tumor size in the shCD133 group after IR and DDP treatment. $* * \mathrm{P}<0.01$.

were compared between HepG2-CD133+ and HepG2-CD133 cells. The present study showed that HepG2-CD133+ cells possess higher proliferation, tumorsphere-forming and colony-forming abilities in vitro and tumorigenesis ability in vivo, which revealed that HepG2-CD133 ${ }^{+}$cells have stem- like features and could be treated as LCSCs. Suetsugu et al (16) demonstrated $\mathrm{CD} 133^{+} \mathrm{HCC}$ cells as cancer stem/progenitor cells, which is consistent with our findings. Thus, CD133 could be regarded as a potential target for stem-targeted therapy for HCC. 


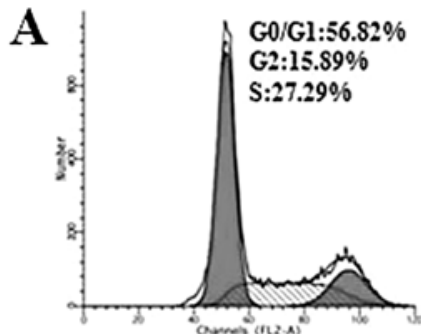

shCD133
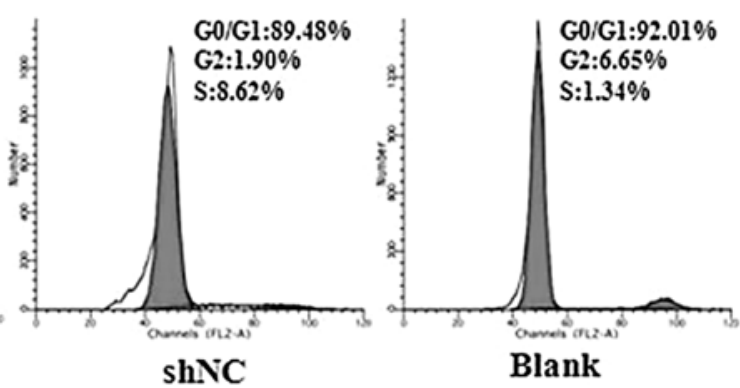
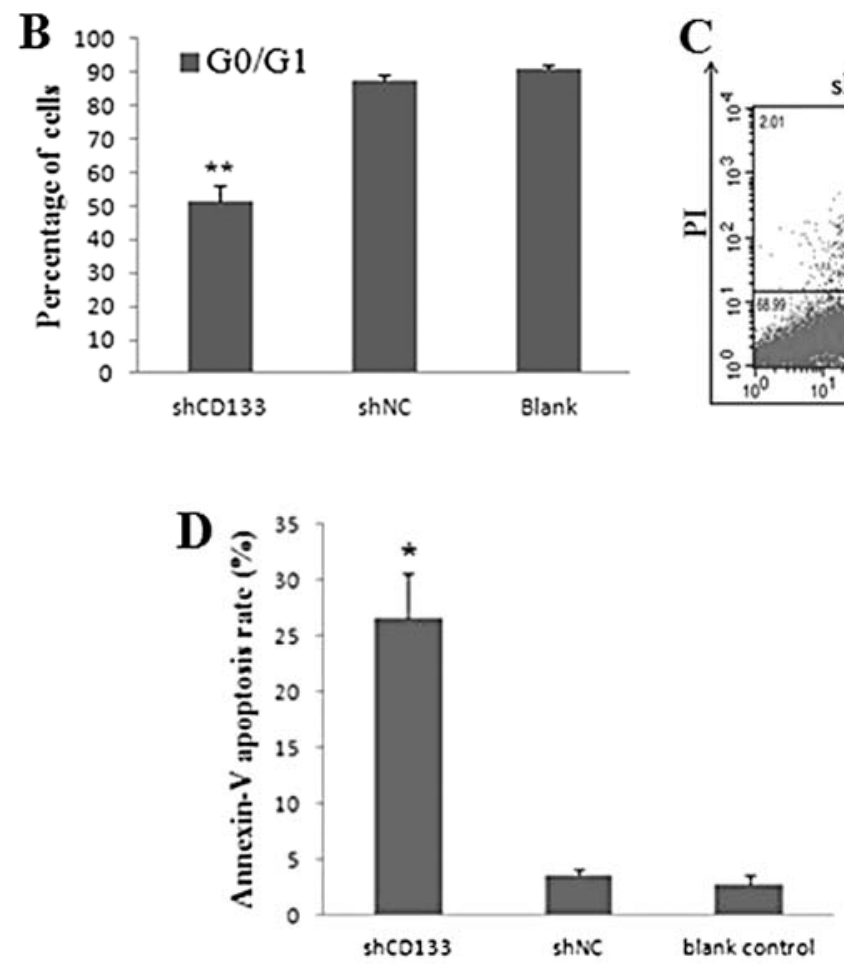

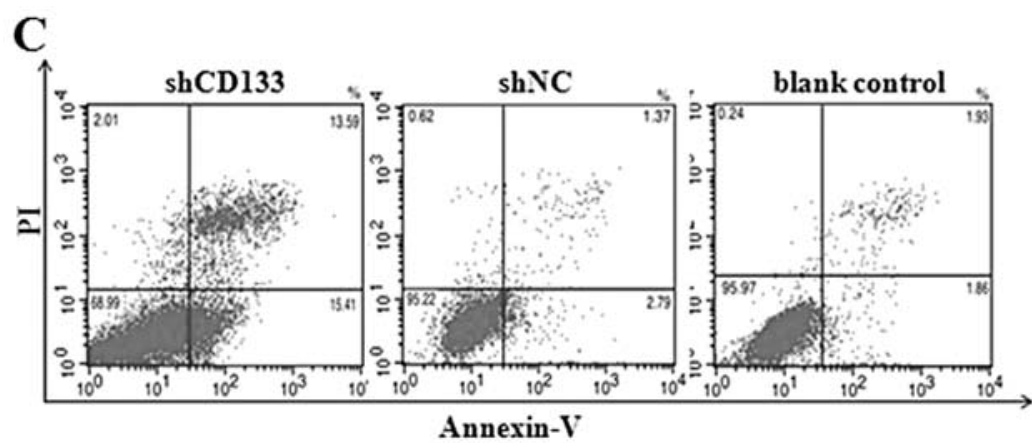

E

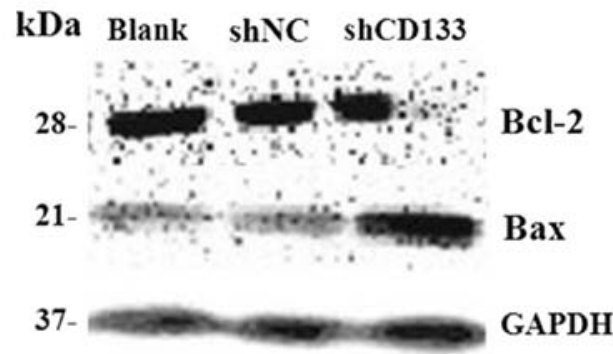

Figure 6. Effects of CD133 downregulation on cell cycle and apoptosis. (A and B) The effect of CD133 downregulation on cell cycle was examined by flow cytometry. G0/G1 phase cells were significantly reduced in the shCD133 group. (C and D) The percentages of apoptotic cells were presented. (E) Western blot analysis on molecules involved in cell survival regulation. ${ }^{*} \mathrm{P}<0.05,{ }^{* *} \mathrm{P}<0.01$.

Next, we infected HepG2-CD133+ cells with lentiviruses containing shRNA targeting CD133. After the silencing of CD133, the cell proliferation, tumorsphere formation and colony formation of LCSCs were significantly inhibited. Furthermore, attenuated tumor formation in NOD/SCID mice was observed after CD133-targeted RNAi, which supported the results of previous studies that reported induced oncogenicity in metastatic melanoma and glioblastoma cells after CD133 downregulation $(37,38)$. However, CD133-silenced LCSCs could still survive in serum-free stem cell medium with decreased tumorsphere formation, compared to sorted CD133- cells which failed to survive in serum-free stem cell medium. Excluding the impacts of CD133 silencing efficiency and experimental errors, one explanation might be that CD133 is not the only molecule maintaining the stemness properties of CD133 ${ }^{+}$ LCSCs. Beyond CD133, the markers of LCSCs proposed at present include CD90, CD44 and OV6 $(39,40)$. Thus, we speculate that there are inner links among these markers expressed in LCSCs and the $\mathrm{CD}_{13} 3^{+}$cells are more likely to express other LCSC markers than CD133- cells.
In addition, we assessed the chemoradiosensitivity of CD133-downregulated LCSCs. Our present study in vitro and in vivo showed that knockdown of CD133 could significantly enhance the sensitivity of LCSCs to chemotherapy and radiotherapy. Cell cycle and apoptosis were then analyzed by flow cytometry to investigate the possible mechanism. Our present results showed that G0/G1 phase LCSCs were dramatically decreased after CD133 silencing by RNAi. Based on the stem cell theory, CSCs are almost exclusively in the G0/G1 phases of the cell cycle (41), and dormant CSCs are more resistant to chemotherapy and radiotherapy than differentiated cancer cells (42). Initiating the LCSCs into differentiation, which may be one of the causes of enhanced sensitivity to traditional treatments in LCSCs. The results of apoptosis analysis showed an increased apoptosis rate after suppression of CD133, and western blotting showed an obviously declined expression of anti-apoptotic protein Bcl-2 and increased expression of apoptosis protein Bax after CD133 downregulation, which may be another cause for enhancing LCSC chemoradiosensitivity. Briefly, these results suggested that enhanced chemoradiosensitivity of LCSCs may be related to altered cell cycle 
distribution and weakened anti-apoptosis ability. However, the molecular mechanisms of CD133 in chemoradiosensitivity remain insufficiently clear and it is necessary to develop further studies on the relationship among LCSC markers.

In summary, downregulation of endogenous CD133 inhibits the stem-like properties and enhances chemoradiotherapeutic response of LCSCs in vitro and in vivo, supporting that CD133 acts as a crucial marker in maintaining the LCSC performances. Stem-targeted therapy via CD133 silencing could be an effective way for the treatment of HCC and potentially for other CD133-expressing cancer types.

\section{Acknowledgements}

This study was supported by Grant no. 81171365 from the National Natural Science Foundation of China. We thank the Chongqing Cancer Institute (Chongqing, China) for providing the electro-linear accelerator.

\section{References}

1. Rich JN: Cancer stem cells in radiation resistance. Cancer Res 67: 8980-8984, 2007.

2. Rosen JM and Jordan CT: The increasing complexity of the cancer stem cell paradigm. Science 324: 1670-1673, 2009.

3. Bao S, Wu Q, McLendon RE, et al: Glioma stem cells promote radioresistance by preferential activation of the DNA damage response. Nature 444: 756-760, 2006.

4. Baumann M, Krause M and Hill R: Exploring the role of cancer stem cells in radioresistance. Nat Rev Cancer 8: 545-554, 2008.

5. Eramo A, Ricci-Vitiani L,Zeuner A, et al: Chemotherapy resistance of glioblastoma stem cells. Cell Death Differ 13: 1238-1241, 2006.

6. Winquist RJ, Boucher DM, Wood M and Furey BF: Targeting cancer stem cells for more effective therapies: Taking out cancer's locomotive engine. Biochem Pharmacol 78: 326-334, 2009.

7. Zhang Q, Shi S, Yen Y, et al: A subpopulation of CD133+ cancer stem-like cells characterized in human oral squamous cell carcinoma confer resistance to chemotherapy. Cancer Lett 289: $151-160,2010$

8. Liu G, Yuan X, Zeng Z, et al: Analysis of gene expression and chemoresistance of $\mathrm{CD} 33^{+}$cancer stem cells in glioblastoma. Mol Cancer 5: 67, 2006.

9. Jin Y, Bin ZQ, Qiang H, et al: ABCG2 is related with the grade of glioma and resistance to mitoantone, a chemotherapeutic drug for glioma. J Cancer Res Clin Oncol 135: 1369-1376, 2009.

10. Chiba T, Kamiya A, Yokosuka O, et al: Cancer stem cells in hepatocellular carcinoma: Recent progress and perspective. Cancer Lett 286: 145-153, 2009.

11. Chiba T, Kita K, Zheng Y, et al: Side population purified from hepatocellular carcinoma cells harbors cancer stem cell-like properties. Hepatology 44: 240-251, 2006.

12. Yin S, Li J, Hu C, et al: CD133 positive hepatocellular carcinoma cells possess high capacity for tumorigenicity. Int J Cancer 120 1444-1450, 2007.

13. Ma S, Chan K, Hu L, et al: Identification and characterization of tumorigenic liver cancer stem/progenitor cells. Gastroenterology 132: 2542-2556, 2007.

14. Yang W, Yan HX, Chen L, et al: Wnt/beta-catenin signaling contributes to activation of normal and tumorigenic liver progenitor cells. Cancer Res 68: 4287-4295, 2008.

15. Yamashita T, Ji J, Budhu A, et al: EpCAM-positive hepatocellular carcinoma cells are tumor-initiating cells with stem/progenitor cell features. Gastroenterology 136: 1012-1024, 2009.

16. Suetsugu A, Nagaki M, Aoki H, et al: Characterization of $\mathrm{CD}_{133^{+}}$ hepatocellular carcinoma cells as cancer stem/progenitor cells. Biochem Biophys Res Commun 351: 820-824, 2006.

17. Yin AH, Miraglia S, Zanjani ED, et al: AC133, a novel marker for human hematopoietic stem and progenitor cells. Blood 90 5002-5012, 1997.
18. Richardson GD, Robson CN, Lang SH, et al: CD133, a novel marker for human prostatic epithelial stem cells. J Cell Sci 117: 3539-3545, 2004.

19. Corbeil D, Roper K, Hellwig A, et al: The human AC133 hematopoietic stem cell antigen is also expressed in epithelial cells and targeted to plasma membrane protrusions. J Biol Chem 275: 5512-5520, 2000 .

20. Uchida N, Buck DW, He D, et al: Direct isolation of human central nervous system stem cells. Pro Natl Acad Sci USA 97: 14720-14725, 2000.

21. Singh SK, Clarke ID, Terasaki M, et al: Identification of a cancer stem cell in human brain tumors. Cancer Res 63: 5821-5828, 2003.

22. Bruno S, Bussolati B, Grange C, et al: $\mathrm{CD} 133^{+}$renal progenitor cells contribute to tumor angiogenesis. Am J Pathol 169: 2223-2235, 2006.

23. O'Brien CA, Pollett A, Gallinger S, et al: A human colon cancer cell capable of initiating tumour growth in immunodeficient mice. Nature 445: 106-110, 2007.

24. Collins AT, Berry PA, Hyde C, et al: Prospective indentification of tumorigenic prostate cancer stem cells. Cancer Res 65: 10946-10951, 2005.

25. Song W, Li H, Tao K, et al: Expression and clinical significance of the stem cell marker CD133 in hepatocellular carcinoma. Int J Clin Pract 62: 1212-1218, 2008.

26. Peichev M, Naiyer AJ, Pereira D, et al: Expression of VEGFR-2 and $\mathrm{AC} 133$ by circulating human $\mathrm{CD} 34(+)$ cells identifies a population of functional endothelial precursors. Blood 95: 952-958, 2000.

27. Xu Y, Wang Z, Wang J, et al: Lentivirus-mediated knockdown of cyclin Y (CCNY) inhibits glioma cell proliferation. Oncol Res 18: 359-364, 2010.

28. Tomuleasa C, Soritau O, Rus-Ciuca D, et al: Isolation and characterization of hepatic cancer cells with stem-like properties from hepatocellular carcinoma. J Gastrointestin Liver Dis 19: 61-67, 2010.

29. Chen YW, Chen KH, Huang PI, et al: Cucurbitacin I suppressed stem-like property and enhanced radiation-induced apoptosis in head and neck squamous carcinoma-derived CD $44^{+} \mathrm{ALDH} 1^{+}$ cells. Mol Cancer Ther 9: 2879-2892, 2010.

30. Shafee N, Smith CR, Wei S, et al: Cancer stem cells contribute to cisplatin resistance in Brca1/p53-mediated mouse mammary tumors. Cancer Res 68: 3243-3250, 2008.

31. Reya T, Morrison SJ, Clarke MF, et al: Stem cell, cancer, cancer stem cells. Nature 414: 105-111, 2001.

32. Bonnet D and Dick JE: Human acute myeloid leukemia is organized as a hierarchy that originates from a primitive hematopoietic cell. Nat Med 3: 730-737, 2007.

33. Clark MF, Dick JE, Dirks PB, et al: Cancer stem cells - perspectives on current status and future directions: AACR workshop on cancer stem cells. Cancer Res 66: 9339-9344, 2006.

34. Mizrak D, Brittan M and Alison MR: CD133: molecule of the moment. J Pathol 214: 3-9, 2008.

35. Zhang J, Luo N, Luo Y, et al: MicroRNA-150 inhibits human CD133-positive liver cancer stem cells through negative regulation of the transcription factor c-MyB. Int J Oncol 40: 747-756, 2012.

36. Korkaya $\mathrm{H}$ and Wicha MS: Selective targeting of cancer stem cells: a new concept in cancer therapeutics. BioDrugs 21: 299-310, 2007.

37. Rappa G, Fodstad O and Lorico A: The stem cell-associated antigen CD133 (promini-1) is a molecular therapeutic target for metastatic melanoma. Stem Cells 26: 3008-3017, 2008.

38. Wang $\mathrm{CH}$, Chiou SH, Chou CP, et al: Photothermolysis of glioblastoma stem-like cells targeted by carbon nanotubes conjugated with CD133 monoclonal antibody. Nanomedicine 7: 69-79, 2011.

39. Yang ZF, Ho DW, Ng MN, et al: Significance of $\mathrm{CD} 90^{+}$cancer stem cells in human liver cancer. Cancer Cell 13: 153-166, 2008.

40. Yang W, Wang C, Lin Y, et al: $\mathrm{OV6}^{+}$tumor-initiating cells contribute to tumor progression and invasion in human hepatocellular carcinoma. J Hepatol 57: 613-620, 2012.

41. Uchida N, Sutton RE, Friera AM, et al: HIV, but not murine leukemia virus, vectors mediated high efficiency gene transfer into freshly isolated G0/G1 human hematopoietic stem cells. Proc Natl Acad Sci USA 95: 11939-11944, 1998.

42. Eyler CE and Rich JN: Survival of the fittest: cancer stem cells in therapeutic resistance and angiogenesis. J Clin Oncol 26: 2839-2845, 2008. 